mesenchymal stem cells to adipogenic (fatproducing), osteogenic (bone-producing) and chondrogenic (cartilage-producing) lineages. These kits provide all the necessary growth factors to direct the differentiation of the mesenchymal stem cells. Millipore also offers differentiation media and kits for neural stem-cell lines and mesenchymal stem cells including a human neuronal-differentiation kit and adipogenesis and osteogenesis kits. Although these kits are available for mesenchymal stem cells, the percentages of differentiated mesenchymal cells can still be low for certain lineages.

\section{Stem cells hitting the clinic?}

Even as culture and differentiation research progresses, the race is starting to bring embryonic stem-cell therapies to the clinic. Novocell of San Diego, California, is searching for a diabetes treatment, exploring the potential of using stem cells for treatment. "Using cadaveric or even fetal cells was a real challenge because you do not have an unlimited source of these cells," says Alan Lewis, chief executive of Novocell. "While developing a delivery system, in the background we were working with embryonic stem cells to derive insulinproducing cells," he says. And that work is starting to pay off because Novocell can derive definitive endoderm from embryonic stem cells and then differentiate these to insulinproducing cells. Novocell is now working to define these insulin-producing cells, while further refining its polyethylene-glycol or PEGbased delivery vehicle that could help to avoid immune-response issues in patients.

As companies move their products closer to clinical trials, the questions on everyone's mind are when and under what conditions will the US Food and Drug Administration (FDA) fire the starter's gun? Advanced Cell Technology and Geron in Menlo Park, California, are currently in discussions with the FDA regarding clinical trials using embryonic stem cellbased therapies for the coming year.

Advanced Cell Technology has developed an application to use embryonic stemcell-derived retinal

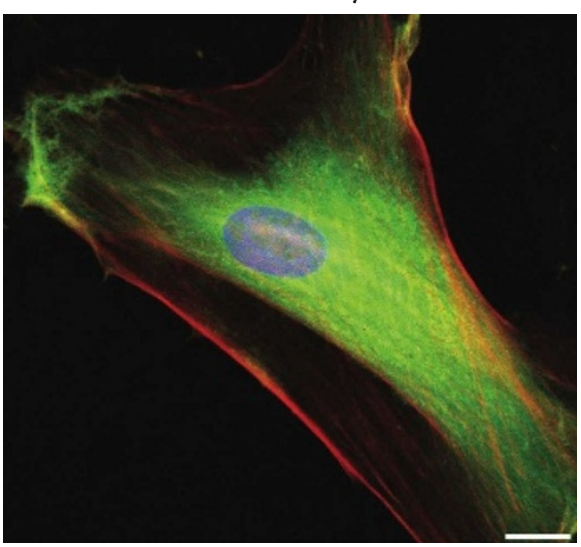

Umbilicial matters: Thermo Fisher Scientific offers adult stem-cell lines derived from Wharton's Jelly. cells thrived in culture. "It turns out that although you can derive cells on human feeders or even feeder-free with extracellular matrices, they do not thrive as well," says Lanza. And for
this reason he thinks that in the long term, when generating large batches of cells for clinical applications, the mouse feeders are currently the optimal method.

It is not yet clear what the first therapy based on embryonic stem cells to reach clinical trials will be, whether it will be using cells raised on MEFs or feeder-free media. And for some researchers it is not pigment epithelium to treat various retinal degenerative diseases such as macular degeneration and retinitis pigmentosa. The company started with this application because in addition to being able to generate large numbers of the required cells, the eye is an immune privilege site, lessening the possibility of an immune response to the cells. Geron has developed a stem cell-based treatment for acute spinal-cord injury. For both of these applications, the companies have utilized stem cells grown with mouse feeder cells. For Advanced Cell Technology this decision was based on how well the stem will be the first pluripotent cells first to reach the clinics (see 'A new path to pluripotency'). George Daley says that it is too early to tell for sure the way stem cell-based therapies will enter the clinics, but he will be watching the developments closely. "I think that it is going to be an exciting time to wait and see," he says.

Nathan Blow is the technology editor for Nature and Nature Methods.

Thomson, J. A. et al. Science 282, 1145-1147(1998).

2. Adewumi, O. et al. Nature Biotechnol. 25, 803-816 (2007)

3. Ludwig, T. E. et al. Nature Biotechnol. 24, 185-187 (2006). even clear if embryonic stem cells themselves

\title{
A NEW PATH TO PLURIPOTENCY
}

In November 2007, two groups headed by James Thompson at the University of Wisconsin-Madison and Shinya Yamanaka at Kyoto University in Japan made headlines when they described methods to reprogramme adult human cells to a pluripotent state. These cells, called induced pluripotent stem (iPS) cells, are genetically modified by the integration of up to four DNA-transcription factors into the adult cell genome. Soon after, in December, George Daley, of Harvard Medical School in Boston, Massachusetts, and his colleagues also demonstrated iPS cells could be generated from a wide variety of adult cells. "For any patient we can use the technique and take a skin biopsy to establish a pluripotent cell," says Daley. One of the most valuable aspects of the iPS-cell technology currently, he says, is the ability to perform disease modelling. And to take advantage of this, Daley and his colleagues

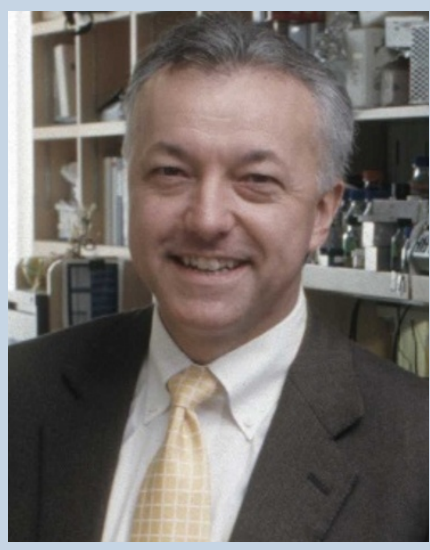

George Daley is exploring the potential of induced pluripotent cells.

are working on generating large numbers of disease specific iPScell lines.

Although an incredible step forward in stem-cell research, iPS cells are in fact at the beginning of a long road. "iPS cells as we make them today are riddled with viruses," says Daley. He says that these viruses can be mutagenic and have the potential to activate oncogenes, so at the moment iPS cells remain a research tool and not a potential therapeutic agent. But the next step for iPS cells could move them closer to therapeutic applications. "One of the next big milestones will be making these cells without the use of viruses - leaving the cells in a genetically pristine state," he says. Robert Lanza from Advanced Cell Technologies in Worcester, Massachusetts agrees and even sees routes to creating iPS cells without genetic modification. "You can potentially use fusion proteins or small molecules - there are many ways to skin the cat here."

But even if iPS cells can be created without genetic modifications, the question that researchers are asking now is, do these cells really have the same properties and potentials as embryonic stem cells? “I am very excited about iPS cells but now we need to look very carefully at the properties of these cell lines," says Martin Pera of the University of Southern California in Los Angeles. Pera says that these cells might differ in their abilities to differentiate in the same way that embryonic stem cells seem to. "If you have to make ten lines for each patient - is patient-specific therapy really realistic or will large banks of iPS cells that are tissue typed be required?"asks Pera.

Only time will tell in what directions iPS cells might be taken for basic research or for clinical applications. And while iPS-cell properties are being studied and new methods to derive these cells without genetic modifications are being created, human embryonic stem-cell research will continue. Pera says that this is the best way to proceed at the moment. "We need to move forward on both fronts." 